\title{
SMOOTHING B-SPLINE ACTIVE CONTOUR FOR FAST AND ROBUST IMAGE AND VIDEO SEGMENTATION
}

\author{
F. Precioso, M. Barlaud \\ Laboratoire I3S - UPRES-A 6070 CNRS \\ Universite de Nice - Sophia Antipolis \\ 2000 route des Lucioles - F-06903 Sophia-Antipolis FRANCE \\ \{Frederic.Precioso, Michel.Barlaud\}@i3s.unice.fr
}

\author{
T. Blu, M. Unser \\ Biomedical Imaging Group, DMT/IOA \\ Swiss Federal Institute of Technology, Lausanne \\ CH-1015 Lausanne EPFL, SWITZERLAND \\ \{Thierry.Blu, Michael.Unser\}@epfl.ch
}

\begin{abstract}
This paper deals with fast image and video segmentation using active contours. Region based active contours using level-sets are powerful techniques for video segmentation but they suffer from large computational cost. A parametric active contour method based on B-Spline interpolation has been proposed in [1] to highly reduce the computational cost but this method is sensitive to noise. Here, we choose to relax the rigid interpolation constraint in order to robustify our method in the presence of noise: by using smoothing splines, we trade a tunable amount of interpolation error for a smoother spline curve. We show by experiments on natural sequences that this new flexibility yields segmentation results of higher quality at no additional computational cost. Hence real time processing for moving objects segmentation is preserved.
\end{abstract}

\section{INTRODUCTION}

We address the problem of image and video segmentation using region-based active contours. The goal of segmentation is to extract image regions corresponding to semantic objects. Image and Video segmentation can be cast in a minimization framework by choosing a criterion which includes region and boundary functionals. Boundary functionals were first proposed by Kass et al.[2] and geodesic active contours by Caselles et al.[3] for active contour segmentation. Region-based active contour were first introduced by Ronfard et al.[4] and Cohen et al.[5]. Then Chan et al.[6], Zhu et al.[7], Amadieu et al.[8], Paragios et al.[9] and Debreuve et al.[10] introduce region-based statistic descriptors for image or video segmentation. Finally Jehan-Besson et al. [11] address the segmentation problem where features of the region to be segmented are involved in region functionals. Gastaud et al. [12] propose a new approach introducing prior shape information in this variational framework as opposed to statistical methods [13] and to (constrained) parametric transformation [14].
All these contour or region-based methods use a levelset approach which is accurate but time consuming.

In this paper, we propose a parametric active contour evolution based on a cubic spline contour [15].

In Section 2, we present a survey of the region-based criterion, the derivation of the criterion and computation of the velocity vector.

In Section 3, we propose a cubic B-spline implementation. Cubic B-splines preserve $C^{2}$ regularity and have excellent approximation properties [16] which means that, for a given accuracy, fewer samples are needed than with other methods; moreover, fast algorithms are available for $\mathrm{B}$-splines, which greatly reduces the computation cost.

Unfortunately, interpolation methods are not robust to noise. This is why we propose to use smoothing splines [17] in the B-spline interpolation approach of [18]. These curves preserve the implementation advantages as the B-splines while softening the interpolation constraint. The relaxation of the interpolation condition is traded for an optimal increase of the smoothness of the spline snake. A smoothness parameter controls the amount of relaxation that is allowed.

In Section 4, we compare the influence of the smoothing spline parameter with the curve-length regularization coefficient. Finally, we show some experiments on real video sequences.

\section{REGION-BASED ACTIVE CONTOURS}

\subsection{Criterion and velocity}

Let us define a general segmentation criterion. For each frame of the sequence, we search a background region $\Omega_{\text {out }}$, and an object regions $\Omega_{i n}$ with a common boundary $\Gamma$ (Fig.1). Thus we minimize a criterion including both region and 


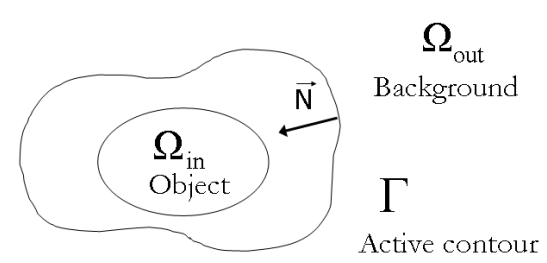

Fig. 1. Domains definition

boundary functionals:

$$
\begin{aligned}
J\left(\Omega_{\text {out }}, \Omega_{\text {in }}, \Gamma\right) & =\int_{\Omega_{\text {out }}} k_{\text {out }}\left(\Omega_{\text {out }}\right) d \sigma \\
& +\int_{\Omega_{\text {in }}} k_{\text {in }}\left(\Omega_{\text {in }}\right) d \sigma+\beta \int_{\Gamma} d s
\end{aligned}
$$

In this criterion, $k_{\text {out }}$ is the descriptor of the unknown background domain $\Omega_{\text {out }}, k_{\text {in }}$ is the descriptor of the unknown object domain $\Omega_{i n}$ and $\beta$ is the weight of the regularization.

Since we use statistical descriptors such as mean, variance or region histogram [19], the descriptors are globally attached to the region $\Omega$.

In the variational Eulerian method proposed by JehanBesson et al. [11] for a region-based active contour segmentation, the authors introduce a dynamical scheme in the criterion. Hence regions become continuously dependent of an evolution parameter $\tau$.

The criterion $J\left(\Omega_{\text {out }}(\tau), \Omega_{\text {in }}(\tau), \Gamma(\tau)\right)$ is denoted by $J(\tau)$. Thus the computation of the derivative provides:

$$
\begin{aligned}
J^{\prime}(\tau) & =\int_{\Omega_{\text {out }}(\tau)} \frac{\partial k_{\text {out }}}{\partial \tau} d \sigma+\int_{\Omega_{\text {in }}(\tau)} \frac{\partial k_{\text {in }}}{\partial \tau} d \sigma \\
& +\int_{\Gamma(\tau)}\left(k_{\text {out }}-k_{\text {in }}-\beta . \kappa+\nabla \beta . \boldsymbol{N}\right)(\boldsymbol{v} \cdot \boldsymbol{N}) d s
\end{aligned}
$$

where $\kappa$ is the curvature of the contour.

The active contour $\Gamma(\tau)$ evolves from an initial position $\Gamma(0)$ towards the object with a velocity $v \boldsymbol{N}$ where $\boldsymbol{N}$ is the inward normal of the active contour:

$$
\begin{cases}\frac{\partial \Gamma(\tau)}{\partial \tau} & =v \boldsymbol{N} \\ \Gamma(0) & =\Gamma_{0}\end{cases}
$$

The velocity expression is deduced from the derivative (2):

$$
v=k_{\text {in }}-k_{\text {out }}+\beta \kappa+\mathbf{A}
$$

The two first terms are global region ones while the third one is a regularization over the contour. A represents some additive local terms which are deduced from the two first region terms in (2). More details (and proofs) are available in $[20,21]$.

\subsection{Implementation}

Region-based active contour evolution can be implemented in two different ways:

- Implicitly, based on the level-set approach [11]. Such a method provides an implicit management of topological changes and yields accurate results, but it suffers from a high computational cost.

- Explicitly, using active parametric contours. Such a method reduces the computational cost substantially and provides a complete control of the data size. The accuracy of the results are dependent on the noise level of the sequence. Using smoothing splines is likely to introduce robustness in this method.

\section{CUBIC SMOOTHING SPLINES}

The curvature term in (4) regularizes the contour. In addition, using an interpolation method reduces the size of the data to handle. We choose cubic spline active contours because cubic splines provide good interpolation accuracy at low computational cost [16] but also because they minimize $\int_{\Gamma(\tau)}\left(\left|x^{\prime \prime}(s)\right|^{2}+\left|y^{\prime \prime}(s)\right|^{2}\right) d s$ under interpolatory constraints [22]; this functional is actually very close to the (squared) curvature $\kappa^{2}$ as shown in [23].

\subsection{Cubic spline Curves}

Cubic B-splines basis functions are $C^{2}$-regular which ensures the existence of the curvature of a cubic spline snake at every point-except, possibly, at cusps.

A curve interpolated with cubic splines is made of a set of $n$ segments, each specified by a polynomial expression of degree 3.

However irregular sampling requires the computation of $n$ different polynomials which is time consuming. In the uniform sampling case, each segment is defined by the same basis functions. Thus, we focus on uniform cubic spline curves.

\subsection{Interpolation or Approximation Splines?}

Our first approach [18] used a uniform cubic spline interpolant to represent the active contour.

The relation between active contour samples $P_{k}=P(k)$ and B-spline coefficients $Q_{k}=Q(k)$ can be written as a convolution:

$$
P(k)=\left(b_{1}^{3} * Q\right)(k)
$$

where $b_{1}^{3}$ is the discrete cubic B-spline kernel.

The inverse convolution operator is defined by:

$$
\left(b_{1}^{3}\right)^{-1}(k) \leftrightarrow B_{1}^{3}(z)^{-1}=\frac{6}{z+4+z^{-1}}
$$


Using the prefiltering approach exposed in [17], this operator is computed very efficiently from a cascade of first order causal and anti-causal recursive filters:

$$
B_{1}^{3}(z)^{-1}=6 \frac{1}{1-z_{s} z^{-1}} \frac{-z_{s}}{1-z_{s} z}
$$

where $z_{s}=-2+\sqrt{3}$.

Hence, the B-spline coefficients of the spline curve that interpolates the data $P(k)$ are given by:

$$
Q(k)=\left(b_{1}^{3}\right)^{-1} * P(k)
$$

In a previous paper, we implemented a region-based active contour segmentation (1) using these curves and we showed that accurate results are obtained in real time [1].

However, interpolation is not robust enough in the presence of noise. This is why we propose to use a less constrained approximation method; namely, the smoothing spline method. The idea, initially proposed by Reinsh [24], is to trade interpolation error for smoothness. Indeed, it is known (Schoenberg [22]) that a cubic spline interpolant minimizes the energy of the second derivative of a function subject to interpolation constraints; instead, Reinsh proposed to minimize a weighted sum of the energy of the second derivative and of the interpolation error at the samples. The result is still a cubic spline, but it does not satisfy anymore the interpolation condition exactly. The interpolation error has been converted into increased smoothness-smaller energy of the second derivative.

Applying the filtering approach [17], we obtain the smoothing spline coefficients from the direct smoothing spline filter:

$S_{\lambda}^{3}(z)^{-1}=\frac{6}{z+4+z^{-1}+6 \lambda\left(z^{-2}-4 z^{-1}+6-4 z+z^{2}\right)}$

This transfert function is factorized into a product of causal and anticausal filters:

$\frac{\left|z_{s}\right|^{2}}{\lambda}\left(\frac{1}{1-2 \operatorname{Re}\left(z_{s}\right) z^{-1}+\left|z_{s}\right|^{2} z^{-2}}\right)\left(\frac{1}{1-2 \operatorname{Re}\left(z_{s}\right) z+\left|z_{s}\right|^{2} z^{2}}\right)$

where $z_{s}$ is the smallest complex root of the characteristic polynomial in the denominator of (9). This prefiltering approach provides an efficient method to compute the smoothing spline coefficients.

The positive parameter $\lambda$ quantifies the tradeoff between interpolation error and smoothness. For $\lambda=0$, no interpolation error is allowed and thus, we are in the case of interpolating splines. When $\lambda$ increases, a larger amount of interpolation error is allowed, hence the spline snake is smoother. We show in the next section that this feature is largely beneficial to the segmentation of noisy images.

\section{EXPERIMENTS}

In this section, we present results obtained for the segmentation of moving objects. Our method is applied to the real "coastguard" video.

The motion-based descriptors for a mobile camera sequence are:

$$
\begin{aligned}
& k_{\text {out }}=\left|I_{n}-\operatorname{Proj}\left(I_{n-1}\right)\right|, \\
& k_{\text {in }}=\text { a positive contant, } \\
& \beta=\text { a positive constant. }
\end{aligned}
$$

The term $\operatorname{Proj}\left(I_{n-1}\right)$ is the projection of the image $I_{n-1}$ onto the referential of image $I_{n}$.

Since these descriptors are region-independent, the evolution equation (3) reduces to:

$$
\frac{\partial \Gamma(\tau)}{\partial \tau}=\left(k_{\text {out }}-k_{\text {in }}-\beta . \kappa\right) \boldsymbol{N}
$$

However, the descrirptor $k_{\text {out }}$ is temporal gradient. Thus this local term is noise sensitive.

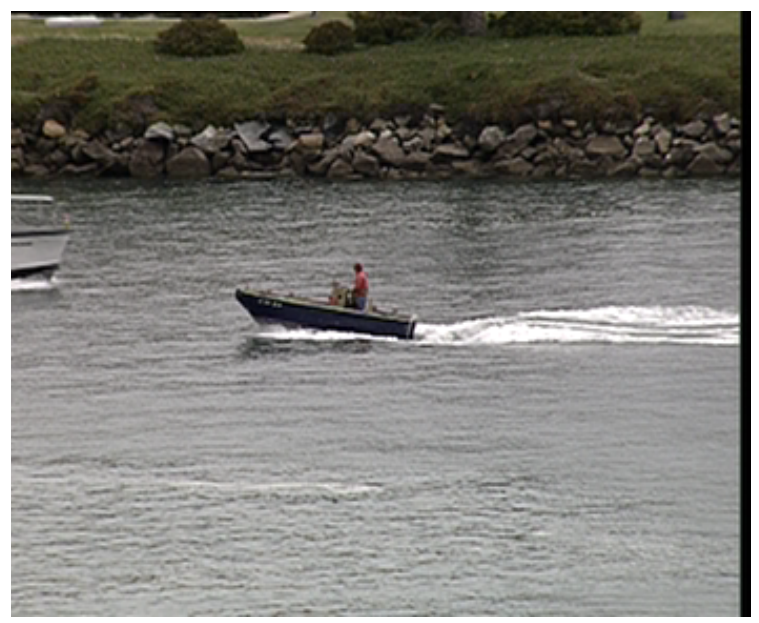

(a) Initial sequence
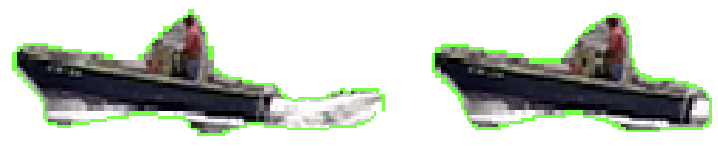

(b) Interpolation $\lambda=0$ and $\beta=20$

(c) Approximation $\beta=0$ and $\lambda=0.5$

Fig. 2. Smoothing spline to smooth contours 
In the "coastguard" sequence Fig.2(a), the wake of the boat behaves like noise for the background descriptor $k_{\text {out }}$. Thus the contour evolution equation is corrupted by noise.

Fig.2(b) shows results using the cubic spline interpolation method [18]. The smoothness of the contour depends only on the contour length regularization parameter $\beta$. However, the foam in the wake of the boat is kept as part of the object.

Fig.2(c) shows results using the new smoothing spline method proposed here. The smoothness of the contour depends only on the smoothing spline parameter $\lambda$. Relaxing the rigid interpolation constraint brings an obvious improvement: the foam is not kept anymore, whereas the object is still reasonably well-segmented.

We can thus say that the smoothing spline method provides global robustness to noise-like data. The accuracy results on a real video sequence show the improvement of our smoothing spline method over a direct regularization of the segmentation criterion.

\section{CONCLUSION}

In this paper, we address here the problem of image and video segmentation by working out a new region-based method using smoothing cubic spline active contours.

Instead of spline interpolation, we have chosen a smoothing spline approximation because we want the method to be more robust in the presence of noise. As a consequence of the very low computational cost of the B-spline implementation, real-time segmentation is achieved. Moreover, the smoothing spline parameter $\lambda$ provides a tunable tradeoff between interpolation error and contour smoothness. It has to be pointed out that the range allowed for this parameter is quite large, which means that it can be chosen a priori for a large set of different sequences.

The accuracy results on a real video sequence show the improvement of our smoothing spline method over a direct regularization of the segmentation criterion.

\section{REFERENCES}

[1] F. Precioso and M. Barlaud, "Regular b-spline active contours for fast video segmentation," in International Conference on Image Processing, Rochester, NY, 2002.

[2] M. Kass, A. Witkin, and D. Terzopoulos, "Snakes: Active contour models," IJCV, vol. 1, pp. 321-332, 1988.

[3] V. Caselles, R. Kimmel, and G. Sapiro, "Geodesic active contours," IJCV, vol. 22, no. 1, pp. 61-79, 1997.

[4] R. Ronfard, "Region-based strategies for active contour models," IJCV, vol. 13, no. 2, pp. 229-251, 1994.

[5] L. Cohen, E. Bardinet, and N. Ayache, "Surface reconstruction using active contour models," in SPIE Conference on Geometric Methods in Computer Vision, San Diego, CA, 1993.
[6] T. Chan and L. Vese, "Active contours without edges," IEEE Transactions on Image Processing, vol. 10, no. 2, pp. 266-277, 2001.

[7] S. Zhu and A. Yuille, "Region competition: unifying snakes, region growing, and bayes/MDL for multiband image segmentation," PAMI, vol. 18, pp. 884-900, 1996.

[8] O. Amadieu, E. Debreuve, M. Barlaud, and G. Aubert, "Inward and outward curve evolution using level set method," in International Conference on Image Processing, Kobe, Japan, 1999.

[9] N. Paragios and R. Deriche, "Geodesic active regions for motion estimation and tracking," in ICCV, Corfu Greece, 1999.

[10] E. Debreuve, M. Barlaud, G. Aubert, and J. Darcourt, "Space time segmentation using level set active contours applied to myocardial gated SPECT," IEEE Transactions on Medical Imaging, vol. 20, no. 7, pp. 643-659, July 2001.

[11] S. Jehan-Besson, M. Barlaud, and G. Aubert, "Dreams: Deformable regions driven by an eulerian accurate minimization method for image and video segmentation. application to face detection in color video sequences," in ECCV, Copenhagen, Denmark, 2002.

[12] M Gastaud, M Barlaud, and G Aubert, "Tracking video objects using active contours and geometric priors," in WIAMIS, London, UK, April 2003, pp. 170-175.

[13] D. Cremers, C. Schnorr, and J. Weickert, "Diffusion-snakes: Combining statistical shape knowledge and image information in a variational framework," in Proceedings of 1st IEEE Workshop on Variational and Level Set Methods in Computer Vision, 2001, pp. 137-144.

[14] Y. Chen, H.D. Tagare, S. Thiruvenkadam, F. Huang, D. Wilson, K.S. Gopinath, R.W. Briggs, and E.A. Geiser, "Using prior shapes in geometric active contours in a variational framework," IJCV, vol. 50, no. 3, pp. 315-328, 2002.

[15] P. Brigger, J. Hoeg, and M. Unser, "B-Spline snakes: A flexible tool for parametric contour detection," IEEE Transactions on Image Processing, vol. 9, no. 9, pp. 1484-1496, September 2000.

[16] P. Thévenaz, T. Blu, and M. Unser, "Interpolation revisited," IEEE Transactions on medical imaging, vol. 19, no. 7, pp. 739-758, July 2000.

[17] M. Unser, A. Aldroubi, and M. Eden, "B-spline signal processing: Part I-theory; part II-efficient design and applications," IEEE Transactions on Signal Processing, vol. 41, no. 2, pp. 821-848, february 1993.

[18] F. Precioso and M. Barlaud, "B-spline active contours with handling of topology changes for fast video segmentation," Eurasip Special issue: Image analysis for multimedia interactive services - PART II, vol. 2002, no. 6, pp. 555-560, June 2002.

[19] G. Aubert, M. Barlaud, O. Faugeras, and S. Jehan-Besson, "Image segmentation using active contours: calculus of variations or shape gradients?," SIAM, 2003.

[20] S. Jehan-Besson, M. Barlaud, and G. Aubert, "Video object segmentation using eulerian region-based active contours," in International Conference on Computer Vision, Vancouver, Canada, October 2001.

[21] S. Jehan-Besson, M. Barlaud, and G. Aubert, "Dreams: Deformable regions driven by an eulerian accurate minimization method for image and video segmentation," IJCV, vol. 53, no. 1, pp. 45-70, June 2003.

[22] I.J. Schoenberg, "Spline functions and the problem of graduation," Proc. Nat. Acad. Sci., vol. 52, pp. 947-950, 1964.

[23] M. Jacob, T. Blu, and M. Unser, "A unifying approach and interface for spline-based snakes," San Diego CA, USA, February 2001, vol. 4322, pp. 340-347, Part I.

[24] C.H. Reinsch, "Smoothing by spline functions," Numer. Math., vol. 10, pp. 177-183, 1967. 